\title{
Design of High-Performance Millimeter Wave and Sub-Millimeter Wave Quasi-Optical Isolators and Circulators
}

\author{
Robert I. Hunter, Duncan A. Robertson, Member, IEEE, Philippe Goy, and Graham M. Smith
}

\begin{abstract}
Faraday rotators using permanently magnetized ferrite materials are used to make quasi-optical isolators and circulators at millimeter wave and sub-millimeter wave frequencies that have far higher performance than their waveguide equivalents. This paper demonstrates state-of-the-art performance for four-port quasi-optical circulators with 60-dB isolation, 0.2-dB insertion loss, and better than 80-dB return loss for devices centered at $94 \mathrm{GHz}$. A method is presented for the accurate characterization of the complex permeability and permittivity of permanently magnetized ferrites via a series of frequency and polarization dependent transmission and reflection measurements. The dielectric and magnetic parameters for the sample are determined by fitting theoretical curves to the measured data. These fitted parameters are then used in a model for a complete quasi-optical Faraday rotator, including matching layers, allowing the accurate design and fabrication of these devices for any specific operational frequency band in the millimeter wave and sub-millimeter wave regime. Examples are given showing typical results and demonstrating how temperature cycling can significantly improve the temperature stability of these devices, while allowing fine tuning of the center frequency. We also indicate the performance possible at higher frequencies to above $1 \mathrm{THz}$ and outline performance of truly planar isolators where lossy polarizer material is built into the Faraday rotator matching structure.
\end{abstract}

Index Terms-Faraday rotator, ferrite, millimeter waves, quasioptics, sub-millimeter waves.

\section{INTRODUCTION}

$\mathbf{T}$ HE FARADAY effect at microwave frequencies is a well-known phenomenon and its description and use in waveguide devices dates back to the seminal works by Polder [1] and Hogan [2]. However, the performance of these waveguide devices, and many waveguide systems, rapidly degrades at high millimeter wave and sub-millimeter wave frequencies. At these frequencies, system designers often prefer to use a mixture of waveguide and low-loss optical techniques for the highest system performance, where millimeter waves are now transmitted as optical beams [3], [4]. However, in many of these

Manuscript received July 19, 2006; revised December 21, 2006. This work was supported by U.K. Engineering and by the Physical Sciences Research Council under a Ph.D. project.

R. I. Hunter, D. A. Robertson, and G. M. Smith are with the Millimetre Wave and High-Field Electron Spin Resonance Group, School of Physics and Astronomy, University of St Andrews, St Andrews, Fife KY16 9SS, U.K. (e-mail: rih1@st-andrews.ac.uk; dar@st-andrews.ac.uk; gms@st-andrews.ac.uk).

P. Goy is with AB Millimetre, 75005 Paris, France (e-mail: abmillimetre@wanadoo.fr).

Digital Object Identifier 10.1109/TMTT.2007.895152

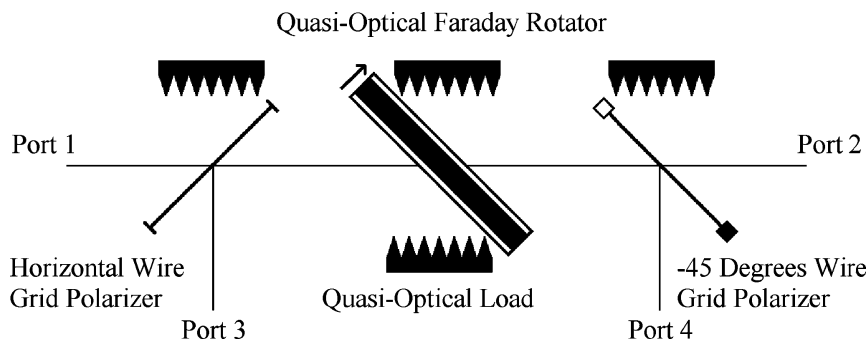

Fig. 1. Schematic diagram of a four-port circulator. A vertically polarized signal entering at port 1 passes through the wire grid polarizer and enters the QOFR where the signal polarization is rotated by $+45^{\circ}$. This signal passes through the second wire grid polarizer and is received at port 2. A signal returning from port 2 passes back through the polarizer grid and into the QOFR, whereupon it is rotated by a further $+45^{\circ}$. This signal is now horizontally polarized and is reflected by the horizontal wire grid polarizer to port 3. In order to construct a three-port circulator or a two-port isolator, the unused ports are terminated with low-reflectivity quasi-optical loads. The QOFR can be angled with respect to the incident signal axis in order to minimize return loss. The direction of magnetization is perpendicular to the planar surface of the rotator, as indicated by the arrow. A typical arrangement at $94 \mathrm{GHz}$ would have an aperture size of $100 \times 100 \mathrm{~mm}$ for both polarizers and ferrite, although smaller (and larger) configurations are possible.

high-frequency quasi-optical system designs, performance is found to by limited by effects related to standing waves between components.

A quasi-optical Faraday rotator (QOFR) is a nonreciprocal polarization rotation device that can be used together with polarizing grids to construct quasi-optical isolators and circulators within quasi-optical circuits [5]. The QOFR itself consists of a layer of ferrite material sandwiched between dielectric impedance matching layers. The ferrite material can be either magnetically soft, in which case external biasing magnets must be used to maintain the necessary magnetization within the ferrite, or the ferrite can be magnetically hard, in which case the device is first saturated in a large field and is then self-biased by the remanent magnetization. The thickness of the ferrite layer is chosen to give $45^{\circ}$ of polarization rotation for a single pass through the device at the desired center frequency. When such a device is placed between angled wire grid polarizers, as shown schematically in Fig. 1, a four-port quasi-optical circulator is produced, which can have exceptionally low insertion loss and return loss, while having excellent isolation properties.

Early QOFRs used magnetically soft ferrite materials and, as a consequence, when operating in a transmission configuration, were limited in aperture by the available external biasing magnets. 
A QOFR of this type operating at $285 \mathrm{GHz}$, and providing more than $-18-\mathrm{dB}$ isolation over a $1 \%$ bandwidth, was reported, with an insertion loss of less than $-2 \mathrm{~dB}$ [6]. This device used a 25.4-mm-diameter soft ferrite disc, externally biased within a samarium-cobalt ring magnet. A transmission QOFR operating at $35 \mathrm{GHz}$ was reported, which gave isolation of greater than $-40 \mathrm{~dB}$ with an insertion loss of less than $-0.1 \mathrm{~dB}$ over $20 \%$ bandwidth, although, again, this was a small area device due to the requirement for external biasing magnets [7].

QOFRs have also been constructed operating in a reflection configuration [8], which allows a biasing magnet to be placed behind the ferrite, easing the aperture size restriction. This configuration is well suited to high-power applications since the reflecting metal backing to the ferrite can be used as a heat sink. Experimental results for this device show comparable performance to the previous $35-\mathrm{GHz}$ transmission devices, with a peak isolation of $-30 \mathrm{~dB}$ and an insertion loss of less than $-0.05 \mathrm{~dB}$ [9].

The use of magnetically hard ferrites was first demonstrated using 30-mm-diameter aperture discs of a sintered hexaferrite (Ferroxdure 330). An isolation of $-17 \mathrm{~dB}$ and an insertion loss of $-1 \mathrm{~dB}$ was reported at $115 \mathrm{GHz}$ [10].

In our own laboratory, we have built and used these devices extensively for many years using a variety of different hexaferrites and matching materials [5], [11]-[17] and they have been an enabling technology for many applications. We have previously constructed $94-\mathrm{GHz}$ QOFRs using $100 \times 100 \mathrm{~mm}$ sintered hexaferrite tiles [11] that have insertion losses from -0.2 to $-0.4 \mathrm{~dB}$ over $20 \%$ bandwidths and have yielded $-60-\mathrm{dB}$ isolation at spot frequencies [12].

One of the advantages of using hexaferrites is that they have a very high crystalline anisotropy field $(\approx 2 \mathrm{~T})$ compared to the maximum demagnetization field $(\approx 0.3 \mathrm{~T})$. This allows large area samples to be magnetized perpendicular to the plane of the material without the use of biasing magnets. Moreover, this magnetization is relatively uniform because the demagnetizing field is constant, to a good approximation, for large area thin devices even approaching the edges of the tile. This removes the restriction on aperture size from biasing magnets and we have constructed robust QOFRs as large as $1000 \times 1000 \mathrm{~mm}$ using plastoferrite materials [13]. This QOFR achieved $-20-\mathrm{dB}$ isolation over a $2-\mathrm{GHz}$ bandwidth, centered at $30.5 \mathrm{GHz}$. The average insertion loss over the whole device at band center was $-0.65 \mathrm{~dB}$. Plastoferrites employ magnetically hard sintered hexaferrite material similar to Ferroxdure 330, which is finely ground and distributed within a rubber binder. This material is rolled or pressed into large sheets. These materials have lower refractive indices than sintered ferrites and are thus easier to match. However, they are usually more lossy and their performance is often limited or complicated by unwanted linear birefringence in the plane of the material, which can limit isolation performance compared to sintered ferrite devices [14], [15]. Nevertheless, devices have been demonstrated at $W$-band with losses as low as $-0.6 \mathrm{~dB}$ and isolations at spot frequencies of $-60 \mathrm{~dB}$, and isolations of better than $-20 \mathrm{~dB}$ over $20-\mathrm{GHz}$ bandwidths [16]. In the 110-180-GHz frequency range, devices with losses of $-2 \mathrm{~dB}$ and greater than $-20-\mathrm{dB}$ isolation across the entire band have been demonstrated. Large area plasto- ferrite QOFRs have also been demonstrated operating below the ferrimagnetic resonance, albeit with restricted bandwidth. In the $10-20-\mathrm{GHz}$ range, $300 \times 300 \mathrm{~mm}$ devices have been constructed with insertion losses of less than $-1.5 \mathrm{~dB}$ over bandwidths of $1-2 \mathrm{GHz}$.

Very large area QOFRs $(645 \times 645 \mathrm{~mm}$ and 265 -mm-diameter circular) have also been constructed operating in the frequency range of $80-100$ and $92-96 \mathrm{GHz}$, respectively [17]. Both of the QOFRs achieved -40-dB isolation at the center frequency and $-20-\mathrm{dB}$ isolation and less than $-1-\mathrm{dB}$ insertion loss across their design bandwidths.

This paper presents new methods for the characterization of hard ferrite materials in their unmagnetized and magnetized states that allow extremely high-performance QOFRs to be designed and optimized for specific frequencies and bandwidths. We present state-of-the-art results for isolators at $W$-band and indicate performance at higher frequencies. This paper also shows how temperature cycling can be used to improve the temperature stability and accurately fine tune the center frequency of devices, and how it is possible to make truly planar isolators where lossy polarizers are built into the matching layers.

\section{THEORY}

The Faraday effect arises due to a difference in the propagation constants for right circularly polarized and left circularly polarized beams transmitted through a material. For the special case where the magnetization is parallel to the direction of propagation, it can be shown that [18]

$$
\begin{aligned}
& \beta^{+}=\omega \sqrt{\epsilon_{0} \epsilon_{r} \mu_{0} \mu_{r}^{+}} \\
& \beta^{-}=\omega \sqrt{\epsilon_{0} \epsilon_{r} \mu_{0} \mu_{r}^{-}}
\end{aligned}
$$

where $\beta^{+}$is the propagation constant for a right-hand, or positive, circularly polarized wave and $\beta^{-}$is that for a left-hand, or negative, circularly polarized wave, $\epsilon_{r}$ is the complex permittivity, and $\mu_{r}^{+}$and $\mu_{r}^{-}$are the complex permeabilities for rightand left-hand circular polarized states. The rotation $\theta$ in radians per unit length is then given by

$$
\theta=\frac{1}{2}\left(\beta^{+}-\beta^{-}\right)
$$

To characterize a ferrite material requires the measurement of $\epsilon_{r}, \mu_{r}^{+}$, and $\mu_{r}^{-}$as a function of frequency. In the millimeter wave and sub-millimeter wave regime, $\epsilon_{r}$ is expected to be independent of magnetization, with the real part being independent of frequency and the imaginary (lossy) part increasing approximately linearly with frequency. This is modeled using an equation of the form

$$
\epsilon_{r}=\epsilon_{r}^{\prime}+i\left(\epsilon_{r 0}^{\prime \prime}+\epsilon_{r f}^{\prime \prime} \cdot f\right) .
$$

In contrast, the real and imaginary parts of $\mu_{r}$ will be strong functions of frequency near resonance. Analytical expressions can be found for $\mu_{r}^{+}$and $\mu_{r}^{-}$for a magnetized ferrite [18], [19]

$$
\begin{aligned}
\mu_{r}^{ \pm^{\prime}} & =1+\frac{f_{m}\left(f_{0} \mp f\right)}{\left(f_{0} \mp f\right)^{2}+f^{2} \alpha^{2}} \\
\mu_{r}^{ \pm^{\prime \prime}} & =\frac{f_{m} f \alpha}{\left(f_{0} \mp f\right)^{2}+f^{2} \alpha^{2}}
\end{aligned}
$$


where $f$ is the frequency and $\alpha$ is a dimensionless damping constant. For a permanently magnetized ferrite, $f_{m}=\gamma M_{R} / 2 \pi$, where $\gamma$ is gyromagnetic ratio and is equal to $28 \mathrm{GHz} / \mathrm{T}$ and $M_{R}$ is the remanent magnetization. The Larmor frequency $f_{0}=$ $\gamma H_{0} / 2 \pi=\left(H_{A}-N_{D} \mu_{0} M_{R}\right) / 2 \pi$, where $H_{0}$ is the effective internal field in the device, $H_{A}$ is the crystalline anisotropy field, and $N_{D}$ is the demagnetization factor, which will be close to unity for a planar device. Thus, if we can determine $f_{0}, f_{m}$, and $\alpha$, we can model $\mu_{r}^{+}$and $\mu_{r}^{-}$at all frequencies. Combined with measurements of $\epsilon_{r}$, we can then model the frequency-dependent single-pass loss and rotation characteristics of a given ferrite. Using standard $A B C D$ transmission matrices for each circular polarization state, we can then model the effects of adding glue and matching layers to get full polarization-dependent $S$-parameters for complete devices over broad frequency ranges.

\section{Characterization Methods}

Our experience of making QOFRs has shown the importance of accurate characterization of hexaferrite materials for highperformance devices. Dielectric and magnetic parameters have been found to vary significantly from ferrite to ferrite. Losses can vary significantly sometimes even within the same batch of material.

The complex permittivity and permeability of materials in the millimeter wave regime has normally been characterized by measurements of the free-space reflection and transmission properties of samples of known thickness as a function of frequency [20]-[27]. Interpretation is relatively straight forward for dielectrics, but more sophisticated models are required for magnetic materials where measurements over extended frequency ranges are required. In [24]-[27], co-polar reflection and transmission measurements were described for ferrites in both their unmagnetized and magnetized states (with the direction of propagation perpendicular to the magnetization axis). Results were then fitted against theoretical equations to determine the various material parameters, such as $\epsilon_{r}, f_{m}$, and the Larmor frequency $f_{0}$.

When the direction of millimeter wave propagation is parallel to the magnetization axis within the ferrite (Faraday rotation), there is the additional complication of linear polarization rotation. A number of methods have been proposed to take this into account. A technique called Faraday angle resonance has been described in [28] in which multiple reflections within a ferrite lead to increased polarization rotation in the ferrite at certain frequencies. By determining the major and minor axes of the transmitted polarization ellipse, the rotation angle is determined as a function of frequency. We have also proposed the use of reflection and transmission measurements of circularly polarized beams to independently characterize the two propagation constants [16]. We have found both methods to be effective, but they tend to be slow and labor intensive as the interferometer has to be reconfigured at each measurement frequency.

In general, we have found that the quickest and most accurate method for the characterization of the magnetic and electrical properties of a ferrite requires reflection and transmission measurements over wide frequency bands for both magnetized

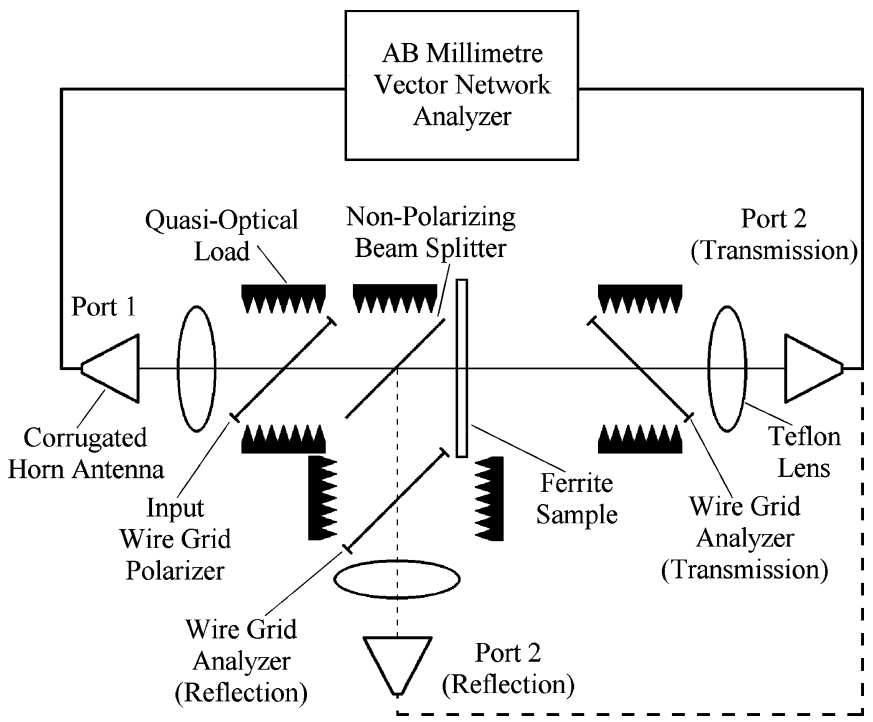

Fig. 2. Block diagram of the quasi-optical measurement system. The dotted line indicates the repositioning of port 2 for reflection measurements. For the characterization of magnetized samples, the corrugated feedhorn at port 2 is rotated to the four principal polarization planes in transmission and the wire grid analyzer is replaced with a suitable wire grid for each polarization plane.

and unmagnetized samples of known thickness. For magnetized samples, we have found it is usually necessary to make measurements for a number of fixed output polarizations to obtain accurate fits for $f_{m}$ and $f_{0}$. A block diagram of our measurement system is shown in Fig. 2. An AB Millimetre vector network analyzer is used to provide tunable coherent radiation from 40 to $167 \mathrm{GHz}$, and over smaller frequency ranges around 300, 400, and $500 \mathrm{GHz}$. Corrugated feedhorns are used to create high-purity Gaussian beams. The samples are plane parallel slabs, which are placed at the focused beamwaist of the Gaussian beams. For transmission measurements of dielectric and nonmagnetized ferrite samples, the analyzer grid and receiving feedhorn are configured for co-polar operation only. The transmitted power is measured with and without the sample in position. In reflection, a nonpolarizing beam splitter is added and the reflected power is measured relative to the reflection from a plane metal mirror placed at the sample position. When measuring samples in reflection, an additional quasi-optical load is placed behind the sample. The resulting transmission and reflection interferograms for the sample are then fitted using a computer model, which treats the sample as a Fabry-Perot etalon to determine $\epsilon$ and $\mu$. This spectrometer has been used to characterize samples of various standard dielectric materials including prospective matching materials, with the results showing excellent agreement with accepted data for these materials [29]. Characterization of samples both in transmission and reflection was found to significantly reduce the uncertainties in both the refractive index and loss measured for the dielectric samples investigated.

Broadband transmission measurements on unmagnetized ferrite samples (from $40 \mathrm{GHz}$ up to $500 \mathrm{GHz}$ ) allow the dielectric properties of the material to be determined away from the ferrimagnetic resonance, where the magnetic losses rapidly become negligible $\left(\mu_{r}^{\prime \prime} \approx 0\right)$ and there are no measurement complications due to the Faraday effect $\left(\mu_{r}^{\prime} \approx 1\right)$. These data are then 
fitted using (4). Measurements near to resonance provide information on $\alpha$ and $f_{0}$. Measurements on magnetized samples then allow $f_{m}, \alpha$, and $f_{0}$ to be determined using (1) and (2) and (5) and (6) together with the dielectric data previously determined from the unmagnetized sample.

For these magnetized measurements, the analyzer grid and receiving feedhorn are reconfigured between transmission measurements to allow the power transmitted to be measured in the four principal polarization planes $\left(0^{\circ}, \pm 45^{\circ}, 90^{\circ}\right)$ relative to a fixed input polarization. Additionally, a nonpolarizing beam splitter is used in the reflection configuration to allow the co-polar and cross-polar reflected power to be measured. The Fabry-Perot etalon model was modified to include the polarization rotation that occurs with each transit through the magnetized ferrite sample. This revised model is then fitted to the six experimentally measured interferograms in order to determine the complex permeability of the sample. The fitting was facilitated by the use of the previously obtained unmagnetized data for the complex permittivity.

\section{DESign of Quasi-Optical Faraday Rotators}

For practical operation, QOFRs require quarter-wavelength matching layers to match the ferrite to free space. To model full devices, it is necessary to multiply together the transfer matrices for the constituent layers in the QOFR (ferrite, dielectric matching, and adhesive) to give the overall transfer matrix for the device. This model can then used to predict the insertion loss, isolation, and return losses.

This model for a complete QOFR has been used to simulate several possible designs and has proven to be an accurate predictor for the performance of finished devices. The model has also been used in a number of simulations to investigate the optimum thicknesses of matching layers of a given dielectric constant to maximize QOFR performance for any given ferrite material.

This is particularly important at $W$-band for the design of high-performance devices, where the complex permeability for one circular polarization state changes relatively rapidly with frequency for hexaferrites. Thus, not only is it impossible to simultaneously impedance match for both circular polarization states at any given frequency, but any impedance match will be a relatively rapidly varying function of frequency. In addition, at $W$-band, the small extra relative loss in power of one circular polarized state compared to the other often needs to be taken into account, as this results in an elliptically polarized beam, which degrades isolation. Any model also needs to take into account the availability of suitable easily machined low-loss dielectrics.

These effects can sometimes be compensated by deliberately mismatching the ferrite by individually fine tuning the thickness of each of the matching layers away from the conventional $\lambda / 4$ thickness. By choosing a dielectric matching material with a permittivity closer to one of the ferrite refractive indices and, therefore, mismatching the other, it is also possible to balance the absorption of left- and right-hand circularly polarized signals and, therefore, reduce the ellipticity of the transmitted linearly polarized signal [29].

This has the effect of increasing the co- and/or cross-polar return losses, but can significantly improve the isolation or the

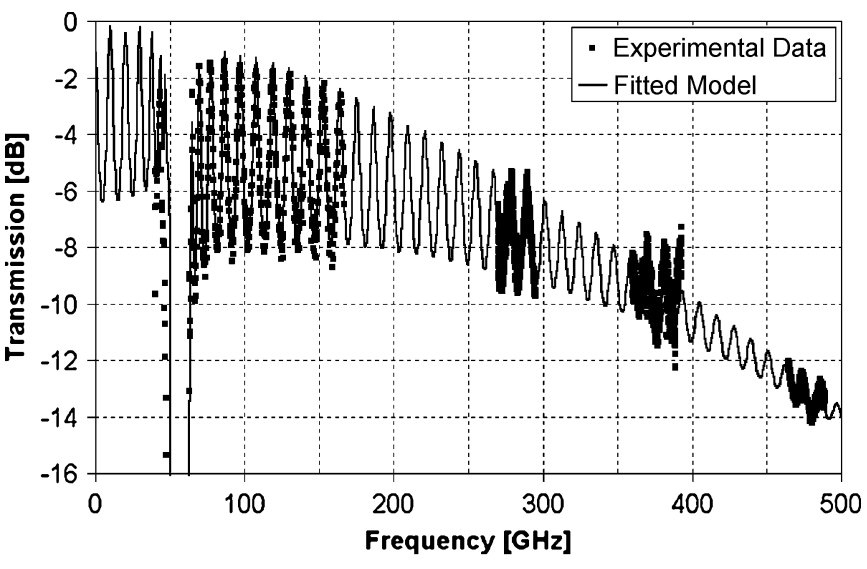

Fig. 3. Transmission spectrum of a 2.97-mm-thick sintered hexaferrite sample and the fitted theoretical model used to determine the dielectric and magnetic parameters.

bandwidth of the QOFR. By this means, it is possible to trade off isolation performance against return loss and insertion loss in order to design a device for a specific application. It is also possible to deliberately mismatch the ferrite in order to increase the operating bandwidth of the QOFR. This reduces the peak isolation from that of a narrow band device, but gives good isolation over the majority of a waveguide band.

Our simulations suggest that, for each ferrite material, there will be a particular optimum permittivity for the matching layers, although it is unlikely that a real dielectric material will be available with both this exact value and the appropriate physical properties. For real devices, it is therefore necessary to make a compromise and select dielectric materials with permittivities close to the ideal value. In order to recover some of the performance lost by not being able to use optimum dielectric matching, the thickness of the matching layers can be fine tuned.

\section{RESULTS}

Preliminary measurements on hexaferrite samples are always made in their unmagnetized state over wide frequency ranges away from the ferrimagnetic resonance to determine the dielectric properties of the ferrite. These do not alter with magnetization and, therefore, these results are of great assistance with the subsequent characterization of these samples after magnetization. A typical transmission spectrum is shown in Fig. 3 showing strong magnetic resonance absorption in the region $45-60 \mathrm{GHz}$ and dielectric losses increasing at higher frequencies.

The characterization of magnetized hexaferrite samples is more difficult since parameters must be found for the model that simultaneously yield good agreement with all measured transmission and reflection spectra for the sample. This fitting process is facilitated by the use of dielectric parameters determined from the unmagnetized characterization and by performing the measurements over as wide a frequency range as possible. Ideally this frequency range should cover the region of the magnetic resonance and should also extend to above $150 \mathrm{GHz}$, where the refractive indices of these hexaferrite materials tend towards their asymptotic values since this greatly facilitates the determination of both $f_{m}$ and $f_{0}$. Experimental 
data and the fitted model for the four principal polarization plane transmission spectra for a typical magnetized hexaferrite sample of $100 \times 100 \mathrm{~mm}$ area are shown in Fig. 4. The fit has been optimized for all four transmission spectra and for the co-polar reflection spectrum (not shown). The magnetic parameters determined from the fit of this hexaferrite sample were $f_{0}=46.0 \mathrm{GHz}, f_{m}=10.1 \mathrm{GHz}$, and $\alpha=0.001$. The dielectric parameters, determined from a previous fit to data from the sample in its unmagnetized state, were $\epsilon_{r}^{\prime}=20.85$, $\epsilon^{\prime \prime}=0.05$, and $\epsilon_{r f}^{\prime \prime}=0.0002$ per GHz. These results are typical for planar sintered ferrite materials, although $f_{0}, f_{m}$, and $\epsilon_{r}$ have been found to vary considerably between different hexaferrite samples.

For sintered hexaferrite samples, $f_{0}$ is usually found to be in the range $43-52 \mathrm{GHz}$, depending on the shape and crystalline anisotropy of the material, $f_{m}$ is typically between $7-11 \mathrm{GHz}$ and $\epsilon_{r}^{\prime}$ is between 18-22. For plastoferrite materials, $f_{0}$ is generally in the range of $44-48 \mathrm{GHz}$ due to the lower anisotropy of these materials, $f_{m}$ is between $5-6 \mathrm{GHz}$ and $\epsilon_{r}^{\prime}$ is typically in the range of 10-12. The parameters determined from such a characterization of a given hexaferrite are then used to design a complete QOFR, together with data from the characterization of the dielectric matching material and adhesive. The principal uncertainties for the practical realization of the design model are the tolerances required for the thicknesses of the adhesive and dielectric matching layers. In practice, over-thickness dielectric matching layers are glued on to the ferrite and these are then machined to the required thickness, as indicated from the design model.

The predicted insertion loss and isolation performance, together with the measured experimental results for a typical finished QOFR using a sintered hexaferrite, are shown in Fig. 5. It should be noted that the spread in the experimental data in Fig. 5(a) is due to small standing waves because the QOFR was measured at normal incidence to the beam. In practice, the QOFR is angled with respect to the beam axis and these effects disappear. The agreement between the predicted design and measured device shown in Fig. 5 is extremely good. This particular device was one of a batch of eight centered at $94 \mathrm{GHz}$, which all gave almost identical performance, showing that the design model provides an excellent indication of the performance attainable from the final device.

\section{LINEAR BIREFRINGENCE EFFECTS}

The design model is equally applicable to both sintered ferrite and plastoferrite QOFR designs. As noted previously, plastoferrite QOFRs tend to suffer from unwanted linear birefringence that arises due to shear forces imposed during the manufacture process. Depending on the magnitude of the linear birefringence, this can severely affect the isolation performance of the device as the effect couples the two circular polarized states together. This effect becomes more severe at higher frequencies. It can be partially ameliorated by ensuring that one of the principal axes associated with the linear birefringence is aligned midway between the input and output polarizations. This only works for one direction and is normally configured to give maximum isolation at the cost of slightly increased insertion loss.
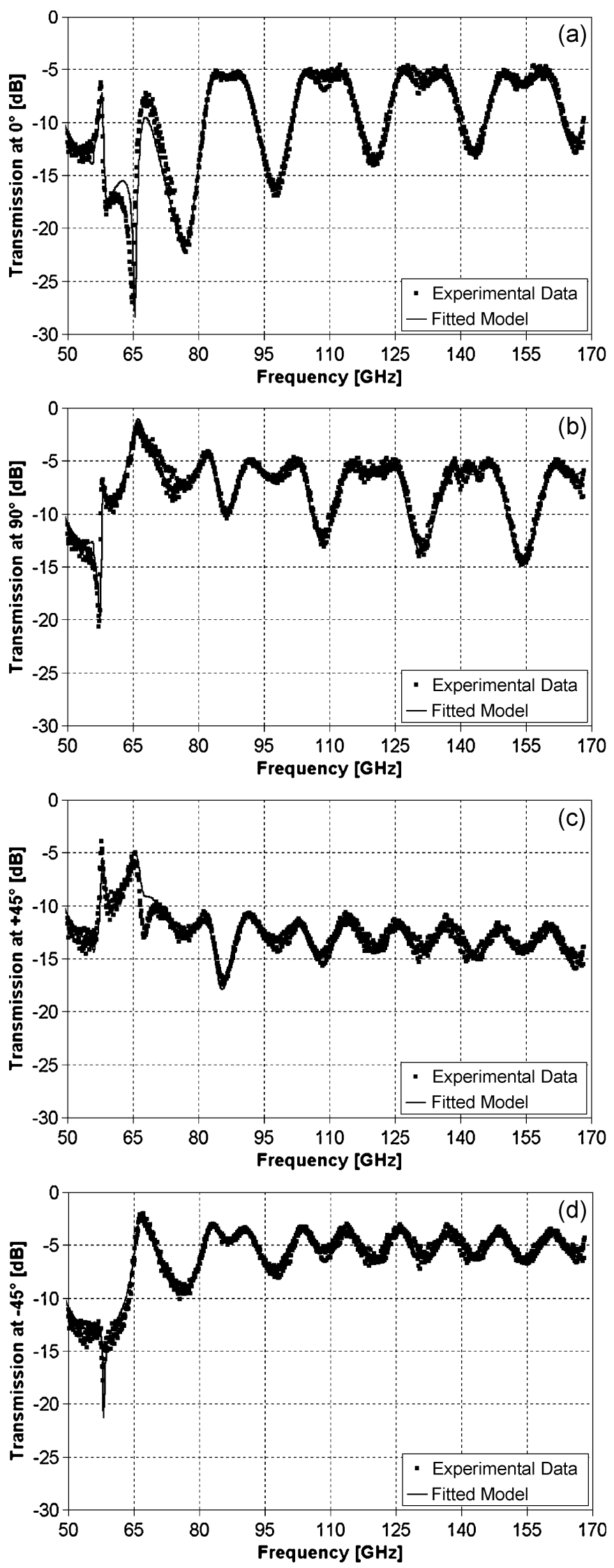

Fig. 4. Transmission spectra for a 1.40-mm-thick sintered hexaferrite sample together with the fitted theoretical model used to determine the dielectric and magnetic parameters for: (a) $+0^{\circ}$, (b) $+90^{\circ}$, (c) $+45^{\circ}$, and (d) $-45^{\circ}$ relative to the input polarization. 

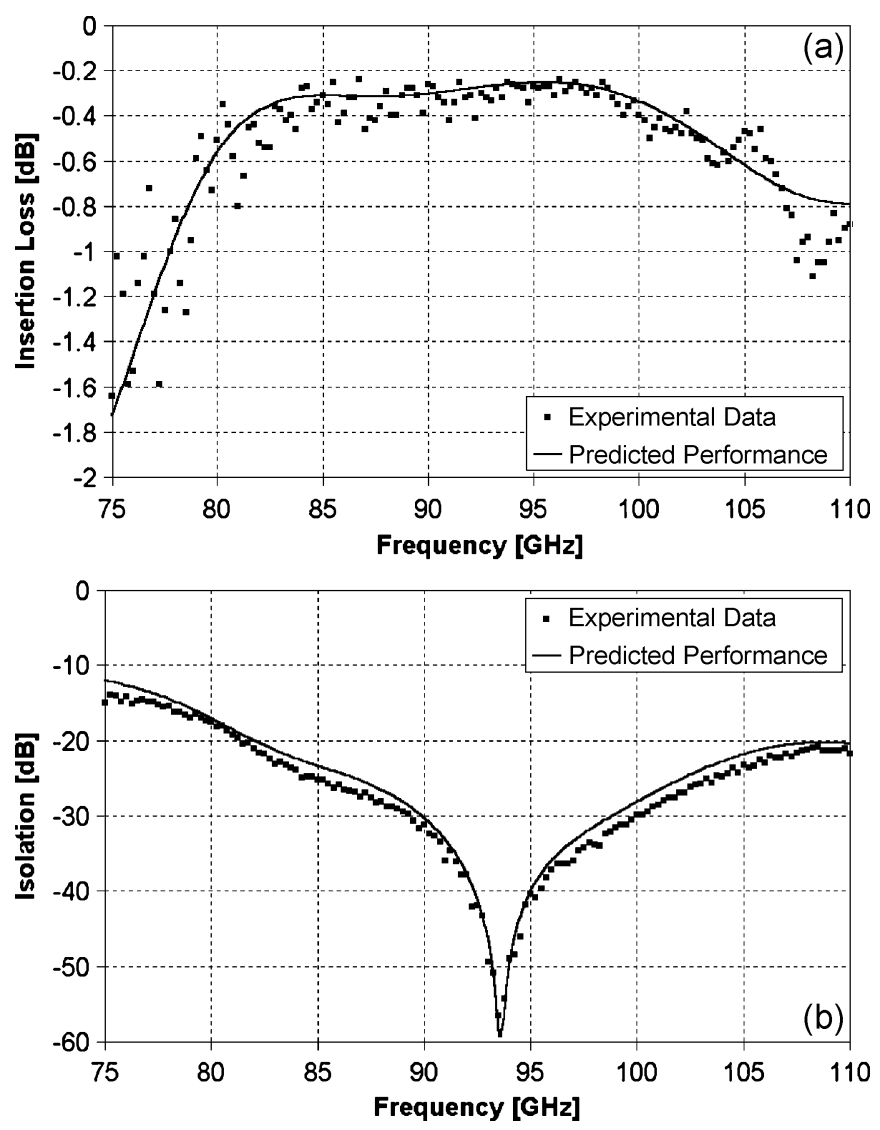

Fig. 5. Predicted and measured spectra showing: (a) insertion loss and (b) isolation for a sintered hexaferrite QOFR designed for a center frequency of $94 \mathrm{GHz}$ using parameters determined by the previous characterization of the ferrite material shown in Fig. 4.

Another way to significantly reduce this problem uses a novel construction method where two layers of plastoferrite material, each of half of the required thickness for $45^{\circ}$ polarization rotation, are glued together such that the roll directions of the two plastoferrite tiles are orthogonal [30].

Fig. 6 shows the predicted and measured insertion loss and isolation for a large area plastoferrite QOFR. Fig. 6(b) also shows experimental data for the best and worst case isolation performance for this device, indicating that although the construction technique does ameliorate the effect of the linear birefringence of the plastoferrite material, it is not entirely suppressed.

\section{RETURN LOSS}

If a QOFR is placed at a Gaussian beam waist, normal to the beam, then the expected and measured return loss at $W$-band is typically -20 to $-25 \mathrm{~dB}$. At higher frequencies, this specification improves as the difference between the two refractive indices associated with the two circular polarization states is reduced. However, we have obtained dramatically improved return loss of up to $-80 \mathrm{~dB}$ over wide frequency ranges by simply angling the QOFR at $45^{\circ}$ and using exceptionally high-performance quasi-optical loads to terminate any power reflected from the Faraday rotator or polarizers. Our measurements of return
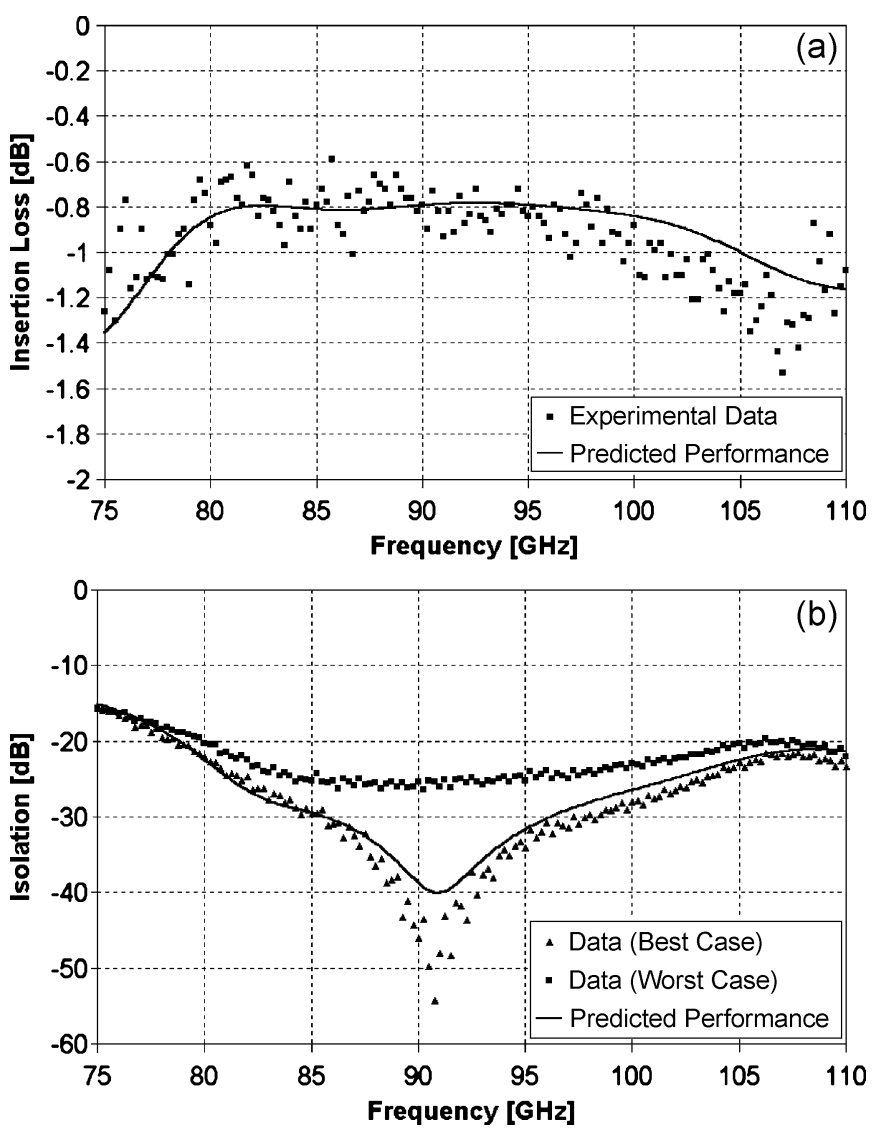

Fig. 6. Predicted and measured spectra showing: (a) insertion loss and (b) isolation for a plastoferrite QOFR designed using parameters determined by previous characterization of the ferrite. Although the patented construction technique reduces the effect of the unwanted linear birefringence of the plastoferrite material, the effect is still evident in (b) where the best and worst case isolation spectra are presented.

loss used calibrated attenuators in conjunction with time-domain reflectometry and heterodyne detection systems, and were dynamic range limited.

This particular optical configuration does not significantly affect the isolation as theory, confirmed by experiment, shows that the Faraday rotation angle is largely insensitive to the angle of incidence of the beam [8] and, in practice, we have still managed to obtain isolations of up to $-60 \mathrm{~dB}$ at spot frequencies.

This extremely low level of return loss is one of the main advantages of quasi-optical isolators/circulators over more conventional waveguide devices where return-loss figures of $-35 \mathrm{~dB}$ over any significant bandwidth would be regarded as impressive.

\section{TEMPERATURE EFFECTS}

For some applications, the effect of temperature variations must be taken into account as these can seriously degrade the performance of some QOFRs. The largest deleterious effect is found on cooling as the coercivity of hexaferrites falls significantly with temperature (near room temperature). As many hexaferrite QOFRs operate near the knee of the demagnetization curve, cooling is thus found to cause a significant decrease in magnetization and, therefore, polarization rotation angle. We have measured up to $10 \%$ changes in magnetization for some 
hexaferrites on cooling from room temperature to $-20^{\circ} \mathrm{C}$. Although the magnetization can be restored by remagnetizing the ferrite this is not always convenient. Conversely, there is a relatively insignificant change in remanent magnetization for most hexaferrites when heated above room temperature, but while keeping well below the Curie temperature.

One method to ameliorate this thermal demagnetization problem is simply to use hexaferrites with a high coercivity relative to their remanent magnetization so that the operating point is well away from the knee of the demagnetization curve. However, these materials do not always have the lowest insertion loss.

Another more attractive solution is to deliberately fabricate a QOFR with a Faraday rotation angle greater than $45^{\circ}$ using a moderate coercivity hexaferrite. The device is magnetized at room temperature, and is then precisely cooled until the desired rotation angle is achieved. This has two major advantages. Firstly, it allows fine temperature tuning of the center frequency of finished QOFRs (where up to $-60-\mathrm{dB}$ isolation may be obtained at spot frequencies) and, secondly, it means the QOFR now becomes much more insensitive to changes in temperature, at least down to the lowest temperature where it was previously tuned.

In practice, we have found this technique of temperature tuning QOFRs has given excellent and reliable performance.

\section{Planar Isolators}

All of the devices described use QOFRs and polarizer grids that are angled with respect to the incident beam, and are used when the highest performance is required for a given system design. However, there are a number of important applications, which do not have sufficient down beam space to implement such schemes, and it becomes desirable to build lossy polarizers into the QOFR itself. For example, it can be useful to build such devices into certain types of antenna structures to prevent local oscillator leakage (e.g., for imaging applications). We have developed a number of methods to make compact lossy polarizers. One method that allows the construction of truly planar isolators is to insert a combination of thin reflecting and absorbing polarizers around the quarter-wavelength matching layers in a Salisbury screen configuration. The lossy polarizer consists of very thin and narrow metallic strips (on a thin Mylar film), whose overall impedance per square characteristic is chosen to give a perfect match at the center frequency, taking into account the impedance of the matching layer.

In practice, at $115 \mathrm{GHz}$, we have obtained better than $-20-\mathrm{dB}$ isolation, with less than $-1-\mathrm{dB}$ insertion loss, over extended bandwidths for a device less than 2.5-mm thick. Detailed modeling has also shown that there is scope to improve this performance further.

\section{DISCUSSION}

The performance of quasi-optical millimeter wave and sub-millimeter wave instrumentation is often limited by standing waves and, therefore, the use of QOFRs has considerable potential to provide high-performance isolation for these systems at frequencies where no suitable alternatives currently exist. At $W$-band, the isolation bandwidth of the device is

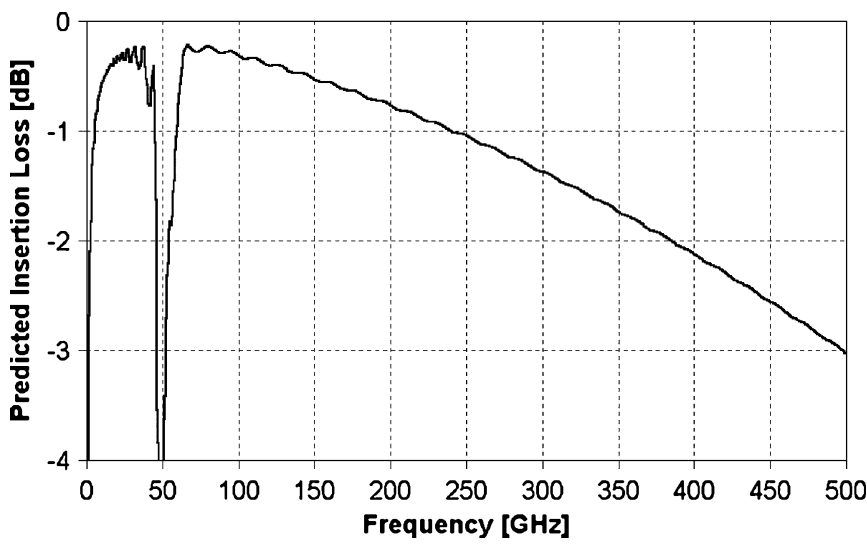

Fig. 7. Predicted optimum attainable spot frequency insertion loss for QOFRs with center frequencies in the range of $0-500 \mathrm{GHz}$. These predictions are based on the characterized parameters for the most suitable available sintered hexaferrite and dielectric matching materials, and these predictions assume that $\lambda / 4$ thick dielectric matching layers are employed at all frequencies.

limited by the relatively rapid change in rotation angle with frequency, as the device is operating relatively close to the ferrimagnetic resonance frequency $f_{0}$. However, well above resonance, the rotation angle becomes frequency independent and the bandwidth becomes limited by the dielectric match. Fig. 7 shows the best predicted insertion loss performance that might be attained from a QOFR with a center frequency in the range $0-500 \mathrm{GHz}$, assuming $\lambda / 4$ thickness matching at each frequency. Modeling also predicts that isolation performance in excess of $-40 \mathrm{~dB}$ would be obtained over the above frequency range. We have validated this at a number of spot frequencies and shows that practical high-performance QOFRs in quasi-optical isolators are feasible to frequencies of $500 \mathrm{GHz}$ and beyond, where no alternatives exist. Indeed, recent measurements using a newly developed terahertz source [31] have shown that the Faraday effect still persists in hexaferrites at frequencies above $1 \mathrm{THz}$. Insertion loss becomes quite severe at these frequencies (more than $-10 \mathrm{~dB}$ ), but such devices may still be useful for some sub-millimeter spectroscopic applications, where measurement accuracy can be more limited by system standing waves than by other signal-to-noise considerations.

It is also possible to construct QOFRs operating below resonance. In this case, the rotation angle decreases fairly rapidly with frequency, meaning that devices become relatively narrow band.

In our own laboratory, we have found QOFRs to be an enabling technology for a wide variety of millimeter wave and submillimeter wave applications. We have used them in millimeter wave spatial filters, frequency filters, imaging systems that allow compact folded optical designs [32], in precision measurement systems such as those for measurement of phase noise [33], and in high-field electron spin resonance spectrometers [34]. QOFRs have also been used in high-power radar systems to provide source isolation [35].

A cascade of several QOFRs is currently under construction for use in a high-power pulsed electron spin resonance spectrometer operating at $94 \mathrm{GHz}$ [36]. This project has a requirement for better than $-90 \mathrm{~dB}$ of source-to-cavity isolation and 
better than $-90-\mathrm{dB}$ return loss. This has now been achieved using a cascade of three QOFRs, together with ultra-low return-loss quasi-optical loads. The insertion loss of the whole cascade is less than $-1 \mathrm{~dB}$. This represents a whole new level of performance for precision millimeter wave systems.

\section{CONCLUSION}

In this paper, we have demonstrated that hexaferrite materials can be accurately characterized to assist in the design of extremely high-performance quasi-optical isolators and circulators, which we believe offer state-of-the-art performance at millimeter wave and sub-millimeter wave frequencies. We believe these devices have a wide variety of applications based on their planar geometry, large area, high power handling, fine tuning, low loss, and high isolation performance.

\section{ACKNOWLEDGMENT}

The authors would particularly like to acknowledge the contributions made to the field of millimeter wave ferrite research at the University of St Andrews, St Andrews, Fife, U.K., by past colleagues Dr. M. R. Webb, Dr. C. P. Unsworth, and Dr. S. Kang.

The authors would also like to thank Prof. D. H. Martin, Queen Mary's College, University of London, U.K., and Dr. R. J. Wylde, Thomas Keating Ltd., Billingshurst, Sussex, U.K., for many useful discussions.

\section{REFERENCES}

[1] D. Polder, "On the theory of ferromagnetic resonance," Philosoph. Mag., vol. 40, pp. 99-115, 1949.

[2] C. L. Hogan, "The ferromagnetic Faraday effect at microwave frequencies and its applications," Rev. Mod. Phys., vol. 25, no. 1, pp. 253-263, Jan. 1953.

[3] J. Lesurf, Millimetre-Wave Optics, Devices \& Systems. Bristol, U.K.: Adam Hilger, 1990.

[4] P. F. Goldsmith, Quasioptical Systems. New York: IEEE Press, 1998.

[5] M. R. Webb, "A mm-wave four port quasi-optical circulator," Int. J. Infrared Millim. Waves, vol. 12, no. 1, pp. 45-63, Jan. 1991.

[6] E. L. Moore, "A $300 \mathrm{GHz}$ quasi-optical Faraday rotation isolator," Int. J. Infrared Millim. Waves, vol. 10, no. 10, pp. 1317-1325, Oct. 1989.

[7] G. F. Dionne, J. A. Weiss, and G. A. Allen, "Nonreciprocal magnetooptics for millimeter waves," IEEE Trans. Magn., vol. 24, no. 6, pp. 2817-2819, Nov. 1988.

[8] B. Lax, J. A. Weiss, N. W. Harris, and G. F. Dionne, "Quasi-optical ferrite reflection circulator," IEEE Trans. Microw. Theory Tech., vol. 41, no. 12, pp. 2190-2197, Dec. 1993.

[9] N. W. Harris, J. A. Weiss, B. Lax, and G. F. Dionne, "The quasi-optical ferrite reflection circulator: Microwave performance and applications," in IEEE MTT-S Int. Microw. Symp. Dig., San Diego, CA, May 1994, pp. $105-108$.

[10] R. J. Wylde, "Gaussian beam-mode circuits for millimetre wavelengths," Ph.D. thesis, Dept. Phys., Queen Mary's College, Univ. London, London, U.K., 1985.

[11] M. R. Webb, "Millimetre wave quasi-optical signal processing systems," Ph.D. thesis, Dept. Phys., Univ. St Andrews, St Andrews, Fife, U.K., 1992.

[12] G. M. Smith, C. P. Unsworth, M. R. Webb, and J. C. G. Lesurf, "Design, analysis and application of high performance permanently magnetized, quasi-optical, Faraday rotators," in IEEE MTT-S Int. Microw. Symp. Dig., San Diego, CA, May 1994, pp. 293-296.

[13] D. A. Robertson, "Very large area Faraday rotators," in Proc. 26th Int. Infrared Millimeter Waves Conf., Toulouse, France, Sep. 2001, pp. 7.8-7.11.

[14] G. M. Smith, S. Kang, C. P. Unsworth, E. Puplett, D. Franklin, and J. C. G. Lesurf, "Microwave, millimeter wave and sub-millimeter wave free-space Faraday rotators," in IEEE MTT-S Int. Microw. Symp. Dig., Orlando, FL, May 1995, pp. 1665-1668.
[15] G. M. Smith, C. P. Unsworth, S. Kang, D. Franklin, and J. C. G. Lesurf, "Design and applications of high frequency quasi-optical Faraday rotators," presented at the 21 st Int. Infrared Millimeter Waves Conf., Berlin, Germany, Jul. 1996, Paper BTh4.

[16] C. P. Unsworth, "A fully automated millimetric rotary polariser quasioptical system," Ph.D. thesis, School Phys. and Astron., Univ. St Andrews, St Andrews, Fife, U.K., 1997.

[17] R. I. Hunter, D. A. Robertson, P. Goy, and G. M. Smith, "Large area $W$-band quasi-optical Faraday rotators for imaging applications," in Proc. 30th Int. Infrared Millimeter Waves Conf., Williamsburg, VA, Sep. 2005, pp. 275-276.

[18] B. Lax and K. J. Button, Microwave Ferrites and Ferrimagnetics. New York: McGraw-Hill, 1962.

[19] A. J. Baden Fuller, Ferrites at Microwave Frequencies. London, U.K.: Peregrinus, 1987.

[20] F. J. Rachford and D. W. Forester, "Characterization of magnetic/dielectric materials at millimeter-wave frequencies," IEEE Trans. Magn., vol. MAG-19, no. 5, pp. 1883-1888, Sep. 1983.

[21] M. N. Afsar, J. R. Birch, and R. N. Clarke, "The measurement of the properties of materials," Proc. IEEE, vol. 74, no. 1, pp. 183-199, Jan. 1986.

[22] F. I. Shimabukuro, S. Lazar, M. R. Chernick, and H. B. Dyson, "A quasi-optical method for measuring the complex permittivity of materials," IEEE Trans. Microw. Theory Tech., vol. MTT-32, no. 7, pp. 659-665, Jul. 1984.

[23] D. K. Ghodgaonkar, V. V. Varadan, and V. K. Varadan, "Free-space measurement of complex permittivity and complex permeability of magnetic materials at microwave frequencies," IEEE Trans. Instrum. Meas., vol. 39, no. 2, pp. 387-394, Apr. 1990.

[24] K. N. Kocharyan, M. N. Afsar, and I. I. Tkachov, "New method for measurement of complex magnetic permeability in the millimeter-wave range-Part II: Hexaferrites," IEEE Trans. Magn., vol. 35, no. 4, pp. 2104-2110, Jul. 1999

[25] — - "Millimeter-wave magnetooptics: New method for characterization of ferrites in the millimeter-wave range," IEEE Trans. Microw. Theory Tech., vol. 47, no. 12, pp. 2636-2643, Dec. 1999.

[26] M. N. Afsar, I. I. Tkachov, and K. N. Kocharyan, "A novel $W$-band spectrometer for dielectric measurements," IEEE Trans. Microw. Theory Tech., vol. 48, no. 12, pp. 2637-2643, Dec. 2000.

[27] M. N. Afsar, K. M. Lee, Y. Wang, and K. N. Kocharyan, "Measurements of complex permittivity and permeability of common ferrimagnets at millimeter waves," IEEE Trans. Magn., vol. 40, no. 4, pp. 2826-2828, Jul. 2004.

[28] M. Raum, "Quasioptical measurement of ferrite material parameters at terahertz frequencies by a new method: Faraday angle resonance," Int. J. Infrared Millim. Waves, vol. 15, no. 7, pp. 1211-1227, Jul. 1994.

[29] R. I. Hunter, “"Characterisation of ferrite and dielectric materials and their use in millimetre-wave quasi-optical devices," $\mathrm{Ph} . \mathrm{D}$. thesis, School Phys. and Astron., Univ. St Andrews, St Andrews, Fife, U.K., 2004.

[30] D. A. Robertson, "Electromagnetic device," U.K. Patent GB2368728B, 2002, Proprietor: Univ. Court, Univ. St Andrews.

[31] T. Edwards, D. Walsh, M. Spurr, C. Rae, M. Dunn, and P. Browne, "Compact source of continuously and widely-tunable terahertz radiation," Opt. Express, vol. 14, pp. 1582-1589, Feb. 2006.

[32] R. Appleby, R. N. Anderton, S. Price, N. A. Salmon, G. N. Sinclair, P. R. Coward, A. R. Barnes, P. D. Munday, M. Moore, A. H. Lettington, and D. A. Robertson, "Mechanically scanned real time passive millimetre wave imaging at $94 \mathrm{GHz}$," in Proc. SPIE 5077, Orlando, FL, Apr. 2003, pp. 1-6.

[33] G. M. Smith and J. C. G. Lesurf, "A highly sensitive millimeter wave quasi-optical FM noise measurement system," IEEE Trans. Microw. Theory Tech., vol. 39, no. 12, pp. 2229-2236, Dec. 1991.

[34] G. M. Smith, J. C. G. Lesurf, R. H. Mitchell, and P. C. Riedi, "Quasioptical CW mm-wave electron spin resonance spectrometer," Rev. Sci. Instrum., vol. 69, no. 11, pp. 3924-3937, Nov. 1998.

[35] B. G. Danly, S. D. Miller, W. Manheimer, A. W. Fliflet, G. Linde, M. Ngo, and W. J. Cheung, "Opportunities for NRL WARLOC radar validation and calibration of NASA Earth observing space missions," in Proc. 29th Int. Infrared Millimeter Waves Conf., Karlsruhe, Germany, Sep. 2004, pp. 807-808.

[36] P. A. S. Cruickshank, D. R. Bolton, D. A. Robertson, D. J. Keeble, and G. M. Smith, "The HIPER project-sub-nanosecond pulse ESR," in Proc. 29th Int. Infrared Millimeter Waves Conf., Karlsruhe, Germany, Sep. 2004, pp. 171-172. 


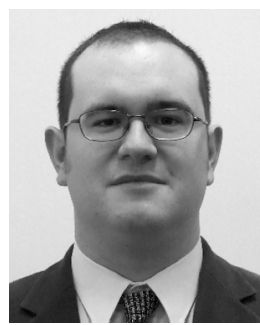

Robert I. Hunter was born in St. Albans, U.K., in 1978. He received the M.Sci. (Hons.) and Ph.D. degrees from the University of St Andrews, St Andrews, Fife, U.K., in 2000 and 2005, respectively.

In 2006, he was a Research Fellow with the Millimetre Wave Group, University of St Andrews, where he was involved with a project to develop a $94-\mathrm{GHz}$ dynamic nuclear polarization (DNP) spectrometer. His research interests include the characterization of ferrites, dielectrics, and absorbing materials, and the development of nonreciprocal ferrite devices for high-performance quasi-optical systems.

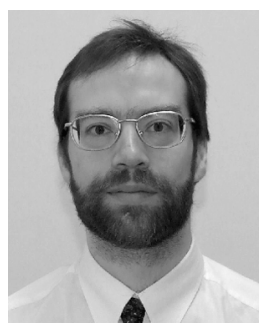

Duncan A. Robertson (S'91-M'94) was born in Aberfeldy, U.K., in 1969. He received the B.Sc. (Hons.) and $\mathrm{Ph} . \mathrm{D}$. degrees from the University of St Andrews, St Andrews, Fife, U.K., in 1991 and 1994, respectively.

From 1994 to 1999, he was with the Millimetre Wave Group, University of St Andrews, under contract to DERA Malvern, where he was involved with battlefield millimeter wave systems for combat identification. From 1999 to 2000, he was a Principal Microwave Engineer with Racal-MESL, Edinburgh, U.K. In 2000, he joined the Photonics Innovation Centre, University of St Andrews, where he was involved with commercializing millimeter-wave technology. In 2004, he rejoined the Millimetre Wave Group, University of St Andrews. He has authored or coauthored over 40 technical papers. He has applied for four patents. His research interests include millimeter-wave radar, radiometry, imaging, electron spin resonance (ESR), materials characterization (ferrites, dielectrics, and absorbers) and antennas.

Dr. Robertson is a Chartered Physicist in the U.K. He is a member of the Institute of Physics.

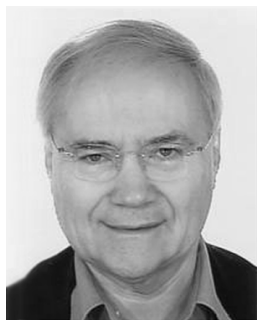

Philippe Goy was born in Charvieu, France, in 1941. He received the Ph.D. degree in physics from the University of Paris, Paris, France, in 1970.

His early academic research was with the Physics Department, Ecole Normale Superieure, Paris, France. In the 1960s and 1970s, he was involved with solid-state physics, particularly on cyclotron resonance in metals with millimeter-submillimeter waves, with the Groupe de Physique des Solides. In the 1970s and 1980s, he was involved with atomic physics, in particular on the interaction of Rydberg atoms with millimeter waves, with the Laboratoire Kastler-Brossel. He has authored or coauthored over 50 papers. In 1989, he was granted a patent for a millimeter-submillimeter vector network analyzer through the Centre National de la Recherche (CNRS). He is responsible for development and applications of this system all over the world, through his company, AB Millimetre.

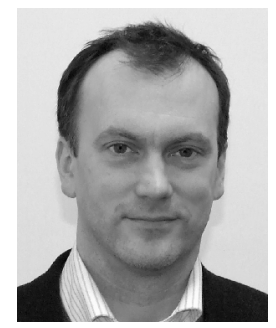

Graham M. Smith was born in Karlsruhe, Germany, in 1963. He received the B.Sc. degree (Hons.) from York University, York, U.K., in 1984, and the M.Sc. and Ph.D. degrees from the University of St Andrews, St Andrews, Fife, U.K., in 1985 and 1990, respectively.

He currently heads the Millimetre Wave and High-Field Electron Spin Resonance (ESR) Group, University of St Andrews, where his research interests primarily focus on the development of millimeter wave components and systems for high-field ESR spectroscopy, DNP, and other applications. 